\title{
DEFORMATIONS OF ELLIPTIC FIBER BUNDLES IN POSITIVE CHARACTERISTIC
}

\author{
HOLGER PARTSCH
}

\begin{abstract}
We study the deformation theory of elliptic fiber bundles over curves in positive characteristics. As applications, we give examples of nonliftable elliptic surfaces in characteristics 2 and 3, which answer a question of Katsura and Ueno. Also, we construct a class of elliptic fibrations, whose liftability is equivalent to a conjecture of Oort concerning the liftability of automorphisms of curves. Finally, we classify deformations of bielliptic surfaces.
\end{abstract}

\section{$\S 1$. Introduction}

In their seminal paper on elliptic surfaces in characteristic $p$, Katsura and Ueno [KU] asked if every elliptic surface of Kodaira dimension 1, over a field of positive characteristic, can be lifted to characteristic 0 . This question is of interest because the known examples of nonliftable surfaces are either quasi-elliptic or of general type. On the other hand, every surface of Kodaira dimension less than or equal to zero is known to be liftable. In this article, we give the first examples of elliptic Kodaira dimension 1 surfaces which are nonliftable.

I briefly sketch how nonliftable elliptic surfaces are constructed. Let $k$ denote an algebraically closed field of characteristic 3. Let $E$ be an elliptic curve over $k$ having $j$-invariant 0 . The automorphism group of such a curve is $G=\mathbb{Z} / 3 \mathbb{Z} \rtimes \mathbb{Z} / 4 \mathbb{Z}$. By $[\mathrm{PS}$, Theorem 7.4] there exists a smooth curve $C$ over $k$ of genus 2 with an étale Galois covering $C^{\prime} \rightarrow C$ of Galois group $G$. Now we set

$$
X=\left(E \times C^{\prime}\right) / G,
$$

where we divide out by the diagonal action of $G$. Note that $X$ has a smooth elliptic fibration $X \rightarrow C$ with a section, coming from the zero section of $E$.

Received May 28, 2010. Revised June 12, 2012. Accepted June 13, 2012.

First published online April 26, 2013.

2010 Mathematics Subject Classification. Primary 14J27; Secondary 14D15.

The author's work was supported by Sonderforschungsbereich/Transregio 45 grant "Periods, moduli spaces and arithmetic of algebraic varieties." 
Clearly, the construction given here is not possible in characteristic 0; there is no elliptic curve over $\mathbb{C}$ with an automorphism group isomorphic to $G$.

To prove that $X$ is in fact not liftable, one classifies deformations of elliptic fiber bundles over smooth curves (e.g., $X \rightarrow C$ ). This suffices, because we will show the following.

Theorem 5.2. If $X \rightarrow C$ is an elliptic fiber bundle of Kodaira dimension 1 , then the unique elliptic fibration extends to every deformation. (That is, the structure of elliptic fiber bundles is preserved under deformations.)

By an elliptic fiber bundle over $C$, we understand an elliptic fibration $X \rightarrow C$ which is locally trivial in the étale topology (see Definition 2.2).

To classify deformations of elliptic fiber bundles, we consider them as torsors over their relative Picard schemes. This is done in Section 4. This way, we will be able to reduce the question of liftability to the case of elliptic fibrations with sections. Here we can use the theory of moduli of elliptic curves. This is the content of Section 3.

In the case of $X$ above, the final result is that $X$ cannot be lifted over a ring $R$ with $3 \neq 0$.

THEOREM 5.6. There exist elliptic fiber bundles in characteristics 2 and 3 that do not lift to characteristic 0 .

Another result is that liftability of a certain class of isotrivial elliptic fibrations would imply the Oort conjecture: given a smooth curve $C$ of higher genus, and a cyclic subgroup $G$ of $\operatorname{Aut}(C)$, then the pair $(C, G)$ can be lifted. Our result is as follows.

TheOrem 5.7. Choose an elliptic curve $E$, and let $G$ act on $E$ by translation. Then the quotient $X=(E \times C) / G$ is liftable if and only if the pair $(C, G)$ is liftable.

As a further application of the theory, we treat the case of bielliptic surfaces. Here we need methods from the deformation theory of abelian schemes. Our main result is the following.

Proposition 6.9. If $X$ is a bielliptic surface, then both elliptic fibrations extend under deformations. In other words, every deformation of a bielliptic surface is bielliptic.

For small $p$, one encounters phenomena which do not appear when considering the same class of surfaces in characteristic 0. For example, deformations become obstructed, which was already observed by Lang [L]. There 
is also the possibility of deforming a Jacobian bielliptic surface into a nonJacobian one, which is absent in characteristic 0 .

\section{§2. Preliminaries}

In this section we introduce some standard techniques which will be used later on. Concerning deformation theory, we follow Schlessinger's fundamental paper [S] in terminology and freely make use of basic facts about pro-representable hulls of deformation functors.

A key problem that will appear in Sections 5 and 6 is of the following form. Given a deformation $\mathcal{X}$ of some scheme $X$, what properties and additional structures carry over to $\mathcal{X}$ ? One example for such properties is étale coverings.

Theorem 2.1 ([Gr2, Théorèmes 5.5 and 8.3]). Let $\mathcal{S}$ be a scheme with a closed subscheme $S_{0}$ having the same topological space as $\mathcal{S}$ itself. Then the functor

$$
\mathcal{X} \mapsto \mathcal{X} \times{ }_{\mathcal{S}} S_{0}
$$

from the category of étale $\mathcal{S}$-schemes to the category of étale $S_{0}$-schemes is an equivalence of categories.

This theorem can be seen as a geometric form of Hensel's lemma from commutative algebra. We note two special cases: the categories of étale Galois covering of $\mathcal{X}$ and $X_{0}$ are equivalent, and so are the categories of finite étale group schemes. Recall that an étale covering $S^{\prime} \rightarrow S$ is called Galois with group $G$ if $G$ acts on $S^{\prime}$ as an $S$-scheme and we have an isomorphism

$$
G \times S^{\prime} \simeq S^{\prime} \times{ }_{S} S^{\prime} \quad \text { given by }(\sigma, x) \mapsto(\sigma(x), x) .
$$

\subsection{Notation}

Finally, let us fix some notation. By $k$ we denote an algebraically closed field of characteristic $p>0$. We denote by $W=W_{\infty}(k)$ its ring of Witt vectors. Let $\mathcal{A l g}_{W}$ be the category of local Artinian $W$-algebras having residue field $k$. Every scheme and every ring will be assumed to be Noetherian, and by a curve over some base scheme $S$ we will always mean a proper smooth and connected 1-dimensional $S$-scheme.

Definition 2.2. Let $S$ be a scheme over some ring $R$. An $R$-morphism $X \rightarrow S$ is called elliptic fiber bundles if there exist a surjective étale morphism $U \rightarrow S$ and an elliptic curve $E$ over $\operatorname{Spec}(R)$ such that

$$
U \times{ }_{S} X \simeq U \times_{\operatorname{Spec}(R)} E .
$$




\section{$\S 3$. Deformations of Jacobian elliptic fiber bundles}

A Jacobian elliptic fiber bundle is a pair $(J / S, \epsilon)$, where $J \rightarrow S$ is an elliptic fiber bundle and $\epsilon$ is a section of $J \rightarrow S$. We can consider $(J / S, \epsilon)$ as an elliptic curve over $S$. In particular, there exists a unique commutative group scheme structure on $J / S$.

We are going to work over an algebra $\Lambda \in \mathcal{A} \lg _{W}$.

Proposition 3.1. Let $\mathcal{S}$ be a proper flat $\Lambda$-scheme such that $\mathcal{S} \otimes_{\Lambda} k$ is integral. Let $\mathcal{J} / \mathcal{S}$ be an elliptic curve over $\mathcal{S}$. For some integer $n \geq 3$ which is prime to $p$, assume that the $n$-torsion subgroup scheme of $\mathcal{J}$ is split; that is, there is an isomorphism

$$
\mathcal{J}[n] \simeq(\mathbb{Z} / n \mathbb{Z})^{2}
$$

Then there exists an elliptic curve $\mathcal{E}$ over $\Lambda$ such that $\mathcal{J}$ is isomorphic to $\mathcal{E} \times{ }_{\Lambda} \mathcal{S}$.

Proof. We can choose a level $n$-structure on $\mathcal{J} / \mathcal{S}$. By [KM, Corollary 4.7.2] we obtain a morphism $c: \mathcal{S} \rightarrow \mathcal{M}_{\Gamma(n)}$ such that $\mathcal{J} \simeq c^{*}\left(\mathcal{E}^{\text {univ }}\right)$, where

$$
\left(\mathcal{E}^{\text {univ }}, \gamma: \mathcal{E}^{\text {univ }}[n] \simeq(\mathbb{Z} / n \mathbb{Z})^{2}\right)
$$

is the universal family of the moduli problem.

Again by $\left[\mathrm{KM}\right.$, Corollary 4.7.2], we know that $\mathcal{M}_{\Gamma(n)}$ is affine. Thus, $c$ factors over the affine hull of $\mathcal{S}$; namely, $\operatorname{Spec}\left(H^{0}\left(\mathcal{S}, \mathscr{O}_{\mathcal{S}}\right)\right)=\operatorname{Spec}(\Lambda)$. Therefore, $\mathcal{J}$ is just the pullback of an elliptic curve $\mathcal{E}$ over $\Lambda$.

In particular, we see that $\mathcal{J} / \mathcal{S}$ is an elliptic fiber bundle under the assumptions of Proposition 3.1. This can be generalized because, for an arbitrary elliptic curve $\mathcal{J} / \mathcal{S}$ and an integer $n$ prime to $p$, we always have that $\mathcal{J}[n]$ is a finite étale group scheme over $\mathcal{S}$, so there exists an étale Galois covering $\mathcal{S}^{\prime} \rightarrow \mathcal{S}$ such that $\mathcal{J}[n] \times \mathcal{S}_{\mathcal{S}} \simeq(\mathbb{Z} / n \mathbb{Z})^{2}$.

Proposition 3.2. Let $\mathcal{S}$ be a proper flat $\Lambda$-scheme such that the special fiber $\mathcal{S} \otimes_{\Lambda} k$ is regular. Let $\mathcal{J} / \mathcal{S}$ be an elliptic curve over $\mathcal{S}$, and let $\mathcal{S}^{\prime} \rightarrow \mathcal{S}$ be a finite étale Galois covering with group $G$ such that $\mathcal{J}[n] \times \mathcal{S} \mathcal{S}^{\prime} \simeq(\mathbb{Z} / n \mathbb{Z})^{2}$ for some $n \geq 3$. Then

$$
\mathcal{J} \simeq\left(\mathcal{E} \times{ }_{\Lambda} \mathcal{S}^{\prime}\right) / G
$$

where $\mathcal{E}$ is an elliptic curve over $\Lambda$, and the action is the diagonal action given by the Galois action on $\mathcal{S}^{\prime}$ and by a homomorphism $G \rightarrow \operatorname{Aut}(\mathcal{E})$ on the left factor. 
Proof. The scheme $\mathcal{S}^{\prime}$ is connected, and because $\mathcal{S} \otimes_{\Lambda} k$ is regular, so is $\mathcal{S}^{\prime} \otimes_{\Lambda} k$. In particular, $\mathcal{S}^{\prime} \otimes_{\Lambda} k$ is integral. Hence, the elliptic curve

$$
\mathcal{J} \times \mathcal{S}^{\prime} \rightarrow \mathcal{S}^{\prime}
$$

satisfies the assumptions of Proposition 3.1. Thus, there exist an elliptic curve $\mathcal{E}$ over $\Lambda$ and an isomorphism $\mathcal{J} \times \mathcal{S}^{\prime} \simeq \mathcal{E} \times{ }_{\Lambda} \mathcal{S}^{\prime}$. In other words, we know that $\mathcal{J}$ and $\mathcal{E} \times{ }_{\Lambda} \mathcal{S}^{\prime}$ are twists of each other, becoming isomorphic under the base change $\mathcal{S}^{\prime} \rightarrow \mathcal{S}$.

Twists of $\mathcal{E} \times{ }_{\Lambda} \mathcal{S}$ are classified up to isomorphism by the Galois cohomology set $H^{1}\left(G, A\left(\mathcal{S}^{\prime}\right)\right)$, where $A$ is the group scheme $\operatorname{Aut}\left(\mathcal{E} \times_{\Lambda} \mathcal{S}\right)$, and we consider its $\mathcal{S}^{\prime}$-valued points as Galois module under $G$.

We claim that the Galois action on $A$ is trivial. We have a closed immersion $A \subset \operatorname{Aut}\left(\mathcal{E}[n] \times{ }_{\Lambda} \mathcal{S}\right)$ by rigidity (see [KM, Corollary 2.7.3]). However, since $\Lambda$ is a strict Henselian ring, we find that $\mathcal{E}[n] \times_{\Lambda} \mathcal{S}$ is the constant group scheme $(\mathbb{Z} / n \mathbb{Z})^{2}$ on $\mathcal{S}$, which in turn implies that $\operatorname{Aut}\left(\mathcal{E}[n] \times{ }_{\Lambda} \mathcal{S}\right)$ is the constant group scheme $\operatorname{GL}(2, \mathbb{Z} / n \mathbb{Z})$ on $\mathcal{S}$.

Finite étale group schemes over $\mathcal{S}$ correspond to finite abstract groups with a continuous $\pi_{1}(\mathcal{S})$-action. We saw that $A$ can be embedded into a group scheme with trivial $\pi_{1}(\mathcal{S})$-action; hence, the action on $A$ has to be trivial as well. The action of $G$ on $A$ is an induced action of a finite quotient $\pi_{1}(\mathcal{S}) \rightarrow G$ and therefore is trivial as well. Thus, we have

$$
H^{1}\left(G, \operatorname{Aut}\left(\mathcal{E} \times{ }_{\Lambda} \mathcal{S}\right)\left(\mathcal{S}^{\prime}\right)\right) \simeq \operatorname{Hom}\left(G, \operatorname{Aut}\left(\mathcal{E} \times{ }_{\Lambda} \mathcal{S}\right)\left(\mathcal{S}^{\prime}\right)\right)
$$

For a homomorphism $\rho$ in the above group, the corresponding twist looks like $\left(\mathcal{E} \times{ }_{\Lambda} \mathcal{S}^{\prime}\right) / G$, where the action of $\sigma \in G$ is given by

$$
(x, y) \mapsto(\rho(\sigma)(x), \sigma y)
$$

Now, let $S \simeq \mathcal{S} \otimes_{R} k$ denote the reduction of $\mathcal{S}$. Given an elliptic curve $E / S$, we can use the above results to give a necessary and sufficient criterion for the existence of Jacobian liftings.

Corollary 3.3. Let $J$ be an elliptic curve over $S$, given by $\left(E \times{ }_{k} S^{\prime}\right) / G$ for some étale Galois covering $S^{\prime} \rightarrow S$ with group $G$ (Proposition 3.2). Denote the action of $G$ on $E$ by $\rho_{0}$. Then there exists a lifting $\mathcal{J} \rightarrow \mathcal{S}$ if and only if there exists a lifting $\mathcal{E}$ of $E$ over $\Lambda$ together with an extension of the action $\rho_{0}$. 
Proof. To show sufficiency is easy. The covering $S^{\prime} \rightarrow S$ lifts uniquely to $\mathcal{S}^{\prime} \rightarrow \mathcal{S}$, which is again Galois with group $G$. If a lifting $\mathcal{E}$ of $E$ with the prescribed properties exists, simply put $\mathcal{J}=\left(\mathcal{E} \times{ }_{\Lambda} \mathcal{S}^{\prime}\right) / G$. This quotient will exist in the category of schemes because $G$ is finite.

In order to show necessity, assume that we have a lifting $\mathcal{J} \rightarrow \mathcal{S}$. Like before, we also have the unique lifting $\mathcal{S}^{\prime} \rightarrow \mathcal{S}$ of the Galois covering. Observe that $\mathcal{J}[n] \times \mathcal{S}_{\mathcal{S}}$ is split, since $\mathcal{J}[n]$ is a finite étale group scheme and the reduction is split by assumption. Using Proposition 3.2, we find that $\mathcal{J} \simeq$ $\left(\mathcal{E} \times{ }_{\Lambda} \mathcal{S}^{\prime}\right) / G$, where the action of $G$ on $\mathcal{E}$ is denoted by $\rho$. We claim that $\rho$ lifts the action $\rho_{0}$.

Consider the induced action of $\rho$ on $\mathcal{E}[n]$ for some integer $n$. The categories of étale group schemes over $k$ and $R$ are equivalent; hence, $\rho$ is determined by its action on the reduction $E[n]$.

For $n \geq 3$ we know that the group homomorphism, given by restricting the automorphism group of an elliptic curve to its scheme of $n$-torsion points, is injective by [KM, Corollary 2.7.2]. However, the isomorphism type of $J[n]$ allows one to determine the action of $G$ on $J[n]$, for it is given by a class in

$$
H^{1}\left(G, \operatorname{Aut}(J[n])\left(S^{\prime}\right)\right) \simeq \operatorname{Hom}\left(G, \operatorname{Aut}(E[n])\left(S^{\prime}\right)\right)
$$

and the element of the latter group which corresponds to $J[n]$ is just $\rho_{0}$. Hence, the restriction of $\rho$ to the reduction has to be $\rho_{0}$.

\subsection{Nonliftable elliptic surfaces}

We postpone the development of the general theory at this point to give some specific examples of Jacobian elliptic fiber bundles which are nonliftable.

3.1.1. Characteristic 3. For the first example, let $k$ be an algebraically closed field of characteristic 3, and let $E$ be an elliptic curve over $k$ with $j$-invariant 0. By [Si, Appendix A, Proposition 1.2], the automorphism group $G$ of $E$ is a semidirect product $\mathbb{Z} / 3 \mathbb{Z} \rtimes \mathbb{Z} / 4 \mathbb{Z}$, where $\mathbb{Z} / 4 \mathbb{Z}$ acts on $\mathbb{Z} / 3 \mathbb{Z}$ in the unique nontrivial way.

As we shall see later on, there exists a curve $C$ over $k$ such that there is a surjection $\pi_{1}(C) \rightarrow G$. Denote by $C^{\prime} \rightarrow C$ the associated finite étale Galois cover. Now we set

$$
J=\left(E \times{ }_{k} C^{\prime}\right) / G
$$

where the action of $G$ on $E$ is the action of the automorphism group. 
Lemma 3.4. Let the characteristic of $k$ be 3 , and let $\Lambda$ be in $\mathcal{A l g} \lg _{W}$. If for an elliptic curve $\mathcal{E}$ over $\Lambda$ the order of the automorphism group of $\mathcal{E}$ is greater than 6 , it follows that $3 \cdot \Lambda=0$.

Proof. Assume by contradiction that the order of $\operatorname{Aut}_{0}(\mathcal{E})$ is greater than 6 . Since 2 is a unit, there is a Weierstrass equation for $\mathcal{E}$ of the form

$$
y^{2}=x^{3}+a_{2} x^{2}+a_{4} x+a_{6}
$$

Admissible transformations look like $x \mapsto u^{2} x+r$ and $y \mapsto u^{3} y+u^{2} s x+t$. The specific form of the equation implies that $t=0$ and $s=0$. Standard arguments show that either $u^{4}=1$ or $u^{6}=1$. Thus, an automorphism group of order $>6$ would have to contain an element of the form $x \mapsto x+r$.

We get an equation $a_{2}=a_{2}+3 r$, which implies that $3 r=0$. But $r$ has to be a unit, for otherwise the reduction map would not be injective on the automorphism group. Thus, $3=0$ follows.

Now we get the following as a direct consequence of Corollary 3.3.

Proposition 3.5. The elliptic bundle $J$ can be lifted (as Jacobian fibration) only over rings in which $3=0$ holds.

3.1.2. Characteristic 2. Now assume that $k$ is an algebraically closed field of characteristic 2. Given an elliptic curve $E$ over $k$ with $j$-invariant 0 , the group of automorphisms will be a semidirect product $G=Q \rtimes \mathbb{Z} / 3 \mathbb{Z}$, where $Q$ is the quaternion group. Similarly to Lemma 3.4, one shows that neither $G$ nor $Q$ can lift to rings with $2 \neq 0$. Now assume the existence of curves $C_{G}$ and $C_{Q}$ over $k$ together with étale Galois covers $C_{G}^{\prime} \rightarrow C_{G}$ with group $G$ and $C_{Q}^{\prime} \rightarrow C_{Q}$ of group $Q$, respectively.

This gives rise to two Jacobian bundles: $J_{G} \simeq\left(C_{G}^{\prime} \times E\right) / G$ and $J_{Q} \simeq$ $\left(C_{Q}^{\prime} \times E\right) / Q$. Again, by Corollary 3.3 we have the following proposition.

Proposition 3.6. The elliptic bundles $J_{G}$ and $J_{Q}$ can be lifted (as Jacobian fibrations) only over rings in which $2=0$ holds.

To finish this discussion, we have to establish the existence of curves over $k$ with specific étale Galois coverings.

To this end, we use a powerful theory which is developed in [PS]. First we fix some group theoretic invariants. Let $G$ be a finite group with the property that the maximal $p$-Sylow subgroup $P$ is normal. We set $H=G / P$. Then one can write $G$ as a semidirect product $P \rtimes H$. 
We denote by $\mathcal{P}$ the maximal elementary abelian quotient of $P$ and consider it as an $\mathbb{F}_{p}$-vector space, which is possible since it is a $p$-torsion group. Let $Z(H)$ be the set of irreducible characters with values in $k$, and let $V_{\chi}$ be an irreducible $k$-representation of $H$ with character $\chi$. On $P$, we have an $H$-action coming from the structure of the semidirect product. This induces an $H$-representation on $\mathcal{P}$. Since $H$ is of order prime to $p$, this representation is semisimple, and we write

$$
\mathcal{P} \otimes_{\mathbb{F}_{p}} k \simeq \oplus V_{\chi}^{m_{\chi}}
$$

The $m_{\chi}$ are thus numerical invariants of the group $G$.

THEOREM ([PS, Theorem 7.4]). Let G be a group having a normal p-Sylow subgroup $P$. Suppose that $H=G / P$ is abelian. Then there exists a curve of genus $g \geq 2$ over $k$ having an étale Galois covering with group $G$ if the minimal number of generators of $H$ is less than or equal to $2 g$, and $m_{\chi} \leq g-1$ holds for every $\chi \in Z(H)$.

In the characteristic 3 example, we had $G=\mathbb{Z} / 3 \mathbb{Z} \rtimes \mathbb{Z} / 4 \mathbb{Z}$. The minimal number of generators of $H=\mathbb{Z} / 4 \mathbb{Z}$ is one; the representation of $H$ on $\mathcal{P}$ is obviously irreducible and given by the sign involution. Thus, the assumptions of the theorem are satisfied for some curve of genus 2 .

In the characteristic 2 examples, we also have that the maximal $p$-Sylow group is normal. Thus, for $g$ sufficiently large, we will find curves with the required coverings.

Later on, we will prove that $J, J_{G}$, and $J_{Q}$ are nonliftable even if we drop any additional requirements on the liftings. This is done in two steps. First, we prove that a lifting in the category of elliptic fiber bundles exists if and only if a Jacobian lifting exists. This is the content of Section 4. The final step is to see that every lifting of an elliptic fiber bundle of Kodaira dimension 1 is an elliptic fiber bundle. This will be done in Section 5 .

\section{§4. Deformations of elliptic torsors}

We start with some general theory on deformations of torsors under smooth commutative group schemes. This mainly rephrases $[\mathrm{ABD}+$, Remarque 9.1.9].

We work in the following setting. Fix a small extension of algebras in $\mathcal{A} \lg _{W}$

$$
0 \rightarrow I \rightarrow \Lambda \rightarrow \Lambda_{0} \rightarrow 0
$$


Let $\mathcal{S}$ be a flat $\Lambda$-scheme, and set $\mathcal{S}_{0}=\mathcal{S} \otimes_{\Lambda} \Lambda_{0}$. We have a closed immersion $i: \mathcal{S}_{0} \rightarrow \mathcal{S}$. Let $\mathcal{G}$ be a smooth commutative $\mathcal{S}$-group scheme, and set $\mathcal{G}_{0}=$ $\mathcal{G} \times \mathcal{S}_{0}$.

For a group functor $\mathcal{F}$ on the category of $\mathcal{S}_{0}$-schemes, we defined the pushforward functor $i_{*} \mathcal{F}$ on $\mathcal{S}$-schemes by sending an $\mathcal{S}$-scheme $\mathcal{T}$ to $\mathcal{F}\left(\mathcal{T} \times \mathcal{S}_{0}\right)$. There is a natural specialization map $s: \mathcal{G} \rightarrow i_{*} \mathcal{G}_{0}$ of group functors. To investigate its kernel, we introduce a coherent sheaf on $\mathcal{S}_{0}$ :

$$
\mathscr{L}=\operatorname{Lie}\left(\mathcal{G}_{0} / \mathcal{S}_{0}\right) \otimes_{\mathcal{O}_{\mathcal{S}_{0}}} I
$$

We have a sequence of group functors on $\mathcal{S}$

$$
0 \rightarrow i_{*} \mathscr{L} \rightarrow \mathcal{G} \stackrel{s}{\rightarrow} i_{*} \mathcal{G}_{0} \rightarrow 0
$$

whose exactness follows from the smoothness of $\mathcal{G}_{0}$, as can be seen affine locally. Taking étale cohomology of (4.1), we obtain the fundamental long exact sequence

$0 \rightarrow i_{*} \mathcal{G}_{0}(\mathcal{S}) / s(\mathcal{G}(\mathcal{S})) \rightarrow H^{1}\left(\mathcal{S}, i_{*} \mathscr{L}\right) \rightarrow H^{1}(\mathcal{S}, \mathcal{G}) \stackrel{s}{\rightarrow}$

$$
\rightarrow H^{1}\left(\mathcal{S}, i_{*} \mathcal{G}_{0}\right) \rightarrow H^{2}\left(\mathcal{S}, i_{*} \mathscr{L}\right)
$$

The sheaves $\mathcal{L}$ and $i_{*} \mathcal{L}$ are coherent modules. We find that

$$
H^{i}\left(\mathcal{S}, i_{*} \mathscr{L}\right) \simeq H^{i}\left(\mathcal{S}_{0}, \mathscr{L}\right) \simeq H_{\mathrm{zar}}^{i}\left(\mathcal{S}, \operatorname{Lie}\left(G_{0} / S_{0}\right)\right) \otimes I
$$

Moreover, we claim that the group $H^{1}\left(\mathcal{S}, i_{*} \mathcal{G}_{0}\right)$ is isomorphic to $H^{1}\left(\mathcal{S}_{0}, \mathcal{G}_{0}\right)$. By the Leray spectral sequence, we get an exact sequence of étale cohomology groups

$$
0 \rightarrow H^{1}\left(\mathcal{S}, i_{*} \mathcal{G}_{0}\right) \rightarrow H^{1}\left(\mathcal{S}_{0}, \mathcal{G}_{0}\right) \rightarrow H^{0}\left(\mathcal{S}_{0}, R^{1} i_{*} \mathcal{G}_{0}\right)
$$

with vanishing last term. It is enough to show that $\left(R^{1} i_{*} \mathcal{G}_{0}\right)_{x}=0$ for every closed point $x$ of $\mathcal{S}$, that is, of $\mathcal{S}_{0}$. By [M, Theorem 1.15], it follows that $\left(R^{1} i_{*} \mathcal{G}_{0}\right)_{x} \simeq H^{1}\left(\operatorname{Spec}\left(\mathscr{O}_{\mathcal{S}_{0}, x}\right), \mathcal{G}_{0}\right)$, and the last group vanishes since $\operatorname{Spec}\left(\mathscr{O}_{\mathcal{S}_{0}, x}\right)$ has only the trivial étale covering. Here, we make use of the assumption that $k$ is algebraically closed.

In our situation, this means the following. Let $J / C$ be a Jacobian elliptic fiber bundle over a curve $C$ over $k$. Let $\mathcal{J}_{0} / \mathcal{C}_{0}$ be a Jacobian lifting of $J / C$ over $\Lambda_{0} \in \mathcal{A l g}{ }_{W}$. 
Proposition 4.1. Let $\mathcal{J} \rightarrow \mathcal{C}$ be a Jacobian lifting of $\mathcal{J}_{0} / \mathcal{C}_{0}$ to $\Lambda$. Then every $\mathcal{J}_{0}$-torsor $\mathcal{X}_{0}$ over $\mathcal{C}_{0}$ lifts to a $\mathcal{J}$-torsor over $\mathcal{C}$. Furthermore, let $m$ be an integer prime to $p$. Then the restriction map

$$
H^{1}(\mathcal{C}, \mathcal{J})[m] \stackrel{\sim}{\rightarrow} H^{1}\left(\mathcal{C}_{0}, \mathcal{J}_{0}\right)[m]
$$

is bijective. This means that liftings of torsors are unique up to p-torsion.

Proof. From the sequence (4.2), we know that the obstruction to lifting the cohomology class associated to $\mathcal{X}_{0}$ lies inside $H^{2}\left(\mathcal{C}_{0}, \operatorname{Lie}\left(\mathcal{J}_{0} / \mathcal{C}_{0}\right)\right) \otimes I$. Note that since $\operatorname{Lie}\left(\mathcal{J}_{0} / \mathcal{C}_{0}\right)$ is a coherent $\mathscr{O}_{\mathcal{C}_{0}}$-module, we can compute its cohomology with respect to the Zariski topology. Since $\mathcal{C}_{0}$ is 1-dimensional, this group is zero.

Once we have lifted the cohomology class, we have to answer the question of whether it is associated to a representable $\mathcal{J}$-torsor. By [R, Lemme XIII], this will be the case if it is torsion. We claim that $H^{1}(\mathcal{C}, \mathcal{J})$ is torsion: since $H^{1}(C, J)$ is torsion, it is enough to show that $H^{1}\left(\mathcal{C}_{0}, \operatorname{Lie}\left(\mathcal{J}_{0} / \mathcal{C}_{0}\right)\right) \otimes I$ is torsion; then the assertion will follow by induction. However, the former group is a $\Lambda$-module, and $\Lambda$ itself is annihilated by some power of $p$.

The second statement now follows directly by taking $m$-torsion in (4.2).

REMARK 4.2. In the case where the base is 0-dimensional, one recovers the well-known fact that the Tate-ŠSafarevič group of an elliptic curve over a complete local ring with algebraically closed residue field is zero, since the first cohomology of the Lie algebra vanishes.

We want to rephrase Proposition 4.1 in the language of deformation functors. For that purpose, we define two deformation functors associated to an elliptic fiber bundle $X \rightarrow C$ over $k$.

Definition 4.3. By a deformation of $X$ over some $\Lambda \in \mathcal{A} \lg _{W}$, we mean a pair $(\mathcal{X}, \epsilon)$, where $\mathcal{X}$ is a flat scheme over $\operatorname{Spec}(\Lambda)$ and $\epsilon$ is an isomorphism $\epsilon: \mathcal{X} \otimes_{\Lambda} k \simeq X$. Let $\mathcal{D}$ ef ${ }_{X}: \mathcal{A} \lg _{W} \rightarrow($ Sets $)$ denote the functor, which sends $\Lambda \in \mathcal{A} \lg _{W}$ to the set of isomorphism classes of deformations of $X / C$.

By a deformation of a fibration $X / C$, we understand a deformation $(\mathcal{X}, \epsilon)$ of $X$ together with a map $\mathcal{X} \rightarrow \mathcal{C}$, such that the isomorphism $\epsilon$ is in fact an isomorphism of $C$-schemes. The functor of deformations of $X$ as fibration is denoted by $\mathcal{F} i b_{X / C}: \mathcal{A l g} \lg _{W} \rightarrow($ Sets $)$.

Two deformations of $\mathcal{X} / \mathcal{C}$ and $\mathcal{X}^{\prime} / \mathcal{C}$ are called isomorphic if there exists an isomorphism of deformations, which is also an isomorphism of $\mathcal{C}$-schemes. 
Let $\left(J / C, e_{0}\right)$ be a Jacobian elliptic fiber bundle. We define the functor $\mathcal{J} a c_{J / C}$ by sending $\Lambda \in \mathcal{A} \lg _{W}$ to a pairs $(\mathcal{J} / \mathcal{C}, e)$, where $\mathcal{J} / \mathcal{C}$ is a deformation of $J / C$ and $e$ is a lift of $e_{0}$. For an element $(\mathcal{J} / \mathcal{C}, e)$ of $\mathcal{J} a c_{J / C}$, notice that a different choice of $e$ leads to an isomorphic element of $\mathcal{J} a c_{J / C}$. Thus, we view $\mathcal{J} a c_{J / C}$ as a subfunctor of $\mathcal{F} i b_{J / C}$, that is, the subfunctor of those deformations admitting a section.

We get a natural map $\mathcal{F} i b_{X / C} \rightarrow \mathcal{J} a c_{J / C}$ as follows. For a deformation $\mathcal{X} / \mathcal{C}$ (not necessarily having a section), we consider the identity component of its Picard scheme. Since $k$ is of characteristic $p$, we can always lift an appropriate $p$ th power of a relatively ample line bundle of $X \rightarrow C$. Therefore, the representability of $\mathrm{Pic}_{X / C}$ follows from the following theorem.

Theorem ([Kl, Theorem 4.8]). Let $Z$ be a projective flat $S$-scheme, having integral geometric fibers. Then $\mathrm{Pic}_{Z / S}$ is representable by a separated S-scheme.

Since $\mathcal{X} / \mathcal{C}$ is an elliptic fiber bundle, the Picard scheme will be smooth, and the identity component $\operatorname{Pic}_{\mathcal{X} / \mathcal{C}}^{0}$ is a smooth elliptic scheme over $\mathcal{C}$. Now $\mathcal{X} / \mathcal{C}$ is in a natural way a torsor under $\operatorname{Pic}_{\mathcal{X} / \mathcal{C}}^{0}$ coming from the isomorphism

$$
\operatorname{Pic}_{\mathcal{X} / \mathcal{C}}^{1} \simeq \mathcal{X} / \mathcal{C}
$$

We define the natural map $\mathcal{F} i b_{X / C} \rightarrow \mathcal{J} a c_{J / C}$ by sending the fibration $\mathcal{X} / \mathcal{C}$ to $\operatorname{Pic}_{\mathcal{X} / \mathcal{C}}^{0}$. In this language, Proposition 4.1 now becomes the first part of our main theorem.

THEOREM 4.4. The map offunctors $\mathcal{F} i b_{X / C} \rightarrow \mathcal{J} a c_{J / C}$ is formally smooth. Moreover, we have

$$
\operatorname{dim}\left(\mathcal{F} i b_{X / C}(k[\epsilon])\right)=\operatorname{dim}\left(\mathcal{J} a c_{J / C}(k[\epsilon])\right)+h^{1}(C, \operatorname{Lie}(J / C)) .
$$

Proof. Recall that $\mathcal{F} i b_{X / C} \rightarrow \mathcal{J} a c_{J / C}$ is formally smooth if for any surjection $\Lambda \rightarrow \Lambda_{0}$ in $\mathcal{A} \lg _{W}$, the induced map

$$
\mathcal{F} i b_{X / C}(\Lambda) \rightarrow \mathcal{F} i b_{J / C}\left(\Lambda_{0}\right) \times_{\mathcal{J} a c_{J / C}}\left(\Lambda_{0}\right) \mathcal{J} a c_{J / C}(\Lambda)
$$

is surjective. By induction, it suffices to verify this for small extensions. However, this follows directly from Proposition 4.1 applied to every element $\mathcal{J}$ of $\mathcal{J} a c_{J / C}(\Lambda)$ with reduction $\mathcal{J}_{0}$ over $\Lambda_{0}$ and a $\mathcal{J}_{0}$-torsor $\mathcal{X}_{0}$.

To prove the statement about the tangent space dimensions, first note that $\mathcal{F} i b_{X / C}$ fulfills the Schlessinger criteria and carries therefore a vector 
space structure on its tangent space. We are going to determine the kernel of the linear map

$$
\mathcal{F} i b_{X / C}(k[\epsilon]) \rightarrow \mathcal{J} a c_{J / C}(k[\epsilon]),
$$

which consists of torsors under $J \otimes k[\epsilon]$. To determine this group, we again use (4.2). The first term vanishes, since every section $C \rightarrow J$ lifts to the trivial deformation. Hence, the kernel is given by $H^{1}(C, \operatorname{Lie}(J / C)) \otimes I$.

\section{$\S 5 . \quad$ Elliptic fiber bundles of Kodaira dimension 1}

We sum up some facts about invertible sheaves associated to elliptic fiber bundles.

Lemma 5.1. Let $f: X \rightarrow C$ be an elliptic fiber bundle over a $k$-curve $C$.

(i) The sheaf $\mathscr{L}=R^{1} f_{*} \mathscr{O}_{X}$ on $C$ is invertible and a torsion element in $\operatorname{Pic}(C)$.

(ii) The relative tangent bundle $\Theta_{X / C}$ is isomorphic to $f^{*} \mathscr{L}$.

(iii) The Kodaira dimension of $X$ equals the Kodaira dimension of $C$.

Proof. We prove (i). To see that $\mathscr{L}$ is invertible, just note that $X \rightarrow C$ has no multiple and hence no wild fibers. To prove that $\mathscr{L}$ is torsion, we proceed as follows. Let $g: J \rightarrow C$ be the Jacobian of $X$, and denote by $e: C \rightarrow J$ the zero section. Since $J=\operatorname{Pic}^{0}(X / C)$, we have

$$
\mathscr{L} \simeq \operatorname{Lie}(J / C) \simeq e^{*} \Theta_{J / C}
$$

(see [LLR, Proposition 1.3]). Thus, it suffices to show that $\Theta_{J / C}$ is torsion in $\operatorname{Pic}(J)$. By Proposition 3.2, we know that there exist both an étale covering $C^{\prime} \rightarrow C$ and an elliptic curve $E$ over $k$ such that $J \times{ }_{C} C^{\prime}$ is isomorphic to $E \times{ }_{k} C^{\prime}$, which we denote by $J^{\prime}$. Let $q$ be morphism $J^{\prime} \rightarrow J$ given by base change. Since $q$ is étale, it follows that $q^{*} \Theta_{J / C} \simeq \Theta_{J^{\prime} / C^{\prime}} \simeq \mathscr{O}_{J^{\prime}}$. Now, we apply the norm map $N: \operatorname{Pic}\left(J^{\prime}\right) \rightarrow \operatorname{Pic}(J)$ (see [Gr1, Section 6.5]), associated to the morphism $q$,

$$
\mathscr{O}_{J} \simeq N\left(q^{*} \Theta_{J^{\prime} / C^{\prime}}\right) \simeq \Theta_{J / C}^{\otimes d},
$$

where $d$ is the degree of $q$. This proves the claim.

For (ii), note that the canonical bundle formula for $X \rightarrow C$ reads

$$
\omega_{X} \simeq f^{*}\left(\mathscr{L}^{-1} \otimes \omega_{C}\right)
$$

The claim follows from the expression

$$
\left(\Theta_{X / C}\right)^{-1} \simeq \Omega_{X / C}^{1} \simeq \omega_{X} \otimes\left(f^{*} \omega_{C}\right)^{-1} .
$$

Finally, (iii) follows from (i) and the canonical bundle formula. 
In particular, we see that an elliptic fiber bundle is of Kodaira dimension 1 if and only if the base curve is of genus greater than or equal to 2 . It is a general fact from the theory of elliptic surfaces that, on a surface of Kodaira dimension 1, there exists exactly one elliptic fibration, given by a suitable power of the canonical sheaf. We generalize this fact to deformations by showing that the unique fibration lifts to an arbitrary deformation of the total space and that this lifting is unique. In other words, we have the following.

Theorem 5.2. For an elliptic fiber bundle $f: X \rightarrow C$ of Kodaira dimension 1 , the forgetful map of deformation functors $\mathcal{F} i b_{X / C} \rightarrow \mathcal{D}$ ef ${ }_{X}$ is an isomorphism.

First, we show injectivity.

Proposition 5.3. Let $f: \mathcal{X} \rightarrow \mathcal{C}$ be a deformation over $\Lambda \in \mathcal{A l g}_{W}$ of an elliptic fiber bundle $f: X \rightarrow C$. Then $f$ is defined by a suitable power of the canonical sheaf $\omega_{\mathcal{X} / \Lambda}$. In particular, $f$ is unique.

Proof. By smoothness of $f$, we get an exact sequence

$$
0 \rightarrow f^{*} \omega_{\mathcal{C} / \Lambda} \rightarrow \Omega_{\mathcal{X} / \Lambda}^{1} \rightarrow \omega_{\mathcal{X} / \mathcal{C}} \rightarrow 0
$$

The outer terms are invertible sheaves. It follows that $\omega_{\mathcal{X} / \Lambda}=f^{*} \omega_{\mathcal{C} / \Lambda} \otimes$ $\Omega_{\mathcal{X} / \mathcal{C}}^{1}$

We claim that $\Omega_{\mathcal{X} / \mathcal{C}}^{1}$ is a torsion element in $\operatorname{Pic}(\mathcal{X})$. Let $f_{0}: X \rightarrow C$ denote the reduction of $f$. By Lemma 5.1(i), we know that the invertible sheaf $\mathscr{L}=R^{1} f_{0 *} \mathscr{O}_{X}$ is a torsion element in $\operatorname{Pic}(C)$. The same is true for $\Omega_{X / C}^{1}$ because $\left(f_{0}^{*} \mathscr{L}\right)^{\wedge} \simeq \Omega_{X / C}^{1}$ (see Lemma 5.1(ii)). We conclude that $\Omega_{\mathcal{X} / \mathcal{C}}^{1}$ is of finite order by induction on the length of $\Lambda$. The tangent space of the deformation functor of invertible sheaves is $H^{1}\left(X, \mathscr{O}_{X}\right)$, which is a $p$-torsion group, since $k$ is of characteristic $p$. So let $n$ denote the order of $\Omega_{\mathcal{X} / \mathcal{C}}^{1}$. For any integer $m$ we get

$$
\omega_{\mathcal{X} / \Lambda}^{\otimes m n} \simeq f^{*}\left(\Omega_{\mathcal{C} / \Lambda}^{1}\right)^{\otimes m n}
$$

Since $\Omega_{\mathcal{C} / \Lambda}^{1}$ is ample on $\mathcal{C}$, we find that $\omega_{\mathcal{X} / \Lambda}$ is semiample on $\mathcal{X}$. Furthermore, $\operatorname{Proj}\left(\operatorname{Sym}\left(\omega_{\mathcal{X} / \Lambda}^{\otimes n}\right)\right)$ is isomorphic to $\operatorname{Proj}\left(\operatorname{Sym}\left(\Omega_{\mathcal{C} / \Lambda}^{1}\right)^{\otimes n}\right) \simeq \mathcal{C}$. This follows because $H^{0}\left(\mathcal{X}, f^{*}\left(\omega_{\mathcal{C} / \Lambda}\right)^{\otimes m n}\right) \simeq H^{0}\left(\mathcal{C},\left(\omega_{\mathcal{C} / \Lambda}\right)^{\otimes m n}\right)$ by the projection formula.

Denote the map $\mathcal{X} \rightarrow \mathcal{C}$ given by the canonical sheaf by $f_{\text {can }}$. On the reduction we find that $f \otimes_{\Lambda} k=f_{0}=f_{\text {can }} \otimes_{\Lambda} k$. However, liftings of $f_{0}$ are 
unique. It is enough to prove this for first-order deformations. The tangent space of the functor of deformations of $f_{0}: X \rightarrow C$ is $H^{0}\left(X^{\prime}, f_{0}^{*} \Theta_{C}\right)$, and this vanishes since the dual $f_{0}^{*} \omega_{C}$ of $f^{*} \Theta_{C}$ has nonzero global sections, because $g(C) \geq 2$.

To prove the surjectivity of $\mathcal{F} i b_{X / C} \rightarrow \mathcal{D} e f_{X}$, we need an estimate for $h^{1}\left(X, \Theta_{X}\right)$.

Lemma 5.4. Denote by $g \geq 2$ the genus of $C$, and set $\mathscr{L}=R^{1} f_{*} \mathscr{O}_{X} \cdot W e$ get

$$
h^{1}\left(X, \Theta_{X}\right) \leq g-1+h^{0}(C, \mathscr{L})+h^{0}\left(C, \mathscr{L}^{2}\right)+3 g-3 .
$$

If $X$ is Jacobian, we get equality in (5.1).

Proof. Since $f$ is smooth, we have an exact sequence

$$
0 \rightarrow \Theta_{X / C} \rightarrow \Theta_{X} \rightarrow f^{*} \Theta_{C} \rightarrow 0
$$

This gives rise to an exact sequence of cohomology groups

$$
H^{1}\left(X, \Theta_{X / C}\right) \rightarrow H^{1}\left(X, \Theta_{X}\right) \rightarrow H^{1}\left(X, f^{*} \Theta_{C}\right) .
$$

Thus, $h^{1}\left(X, \Theta_{X}\right) \leq h^{1}\left(X, f^{*} \Theta_{C}\right)+h^{1}\left(X, \Theta_{X / C}\right)$.

By Lemma 5.1, we have $\Theta_{X / C} \simeq f^{*} \mathscr{L}$. To compute $h^{1}\left(X, \Theta_{X / C}\right)$, we use the Leray spectral sequence and the isomorphisms given by projection formula $\left(f_{*} \Theta_{X / C} \simeq f_{*} f^{*} \mathscr{L} \simeq \mathscr{L}\right.$ and $\left.R^{1} f_{*} \mathscr{L} \simeq \mathscr{L}^{\otimes 2}\right)$ to obtain

$$
0 \rightarrow H^{1}(C, \mathscr{L}) \rightarrow H^{1}\left(X, \Theta_{X / C}\right) \rightarrow H^{0}\left(C, \mathscr{L}^{\otimes 2}\right) \rightarrow 0
$$

By the Riemann-Roch theorem, we get $h^{1}(C, \mathscr{L})=g-1+h^{0}(C, \mathscr{L})$. Thus,

$$
h^{1}\left(X, \Theta_{X / C}\right)=g-1+h^{0}(C, \mathscr{L})+h^{0}\left(C, \mathscr{L}^{\otimes 2}\right) .
$$

For $h^{1}\left(X, f^{*} \Theta_{C}\right)$, we obtain with the same approach and using $\Theta_{C} \simeq$ $f_{*} f^{*} \Theta_{C}$ the sequence

$$
0 \rightarrow H^{1}\left(C, \Theta_{C}\right) \rightarrow H^{1}\left(X, f^{*} \Theta_{C}\right) \rightarrow H^{0}\left(C, \mathscr{L} \otimes \Theta_{C}\right) \rightarrow 0 .
$$

Since $g>1$, the last term vanishes, and we get $h^{1}\left(X, f^{*} \Theta_{C}\right)=3 g-3$.

In the Jacobian case, let $s: C \rightarrow X$ denote the section. The natural map $f^{*} \Omega_{C}^{1} \rightarrow \Omega_{X}^{1}$ has a global splitting given locally by $d(g) \mapsto d\left(s^{*} g\right) \otimes 1$. Dualizing yields a splitting of (5.2). 
Now, we are all set to show the surjectivity of the inclusion $\mathcal{F} i b_{X / C} \rightarrow$ Def $_{X}$.

Proposition 5.5. Let $\Lambda$ be an object of $\mathcal{A l g}_{W}$. Every deformation $\mathcal{X} \in$ $\operatorname{Def}_{X}(\Lambda)$ of the total space of $X$ admits a lifting of the fibration on $X$; in other words, $\mathcal{X} \in \mathcal{F} i b_{X / C}(\Lambda)$.

Proof. Denote by $J$ the Jacobian of $X$. By Proposition 3.2, we know that there is an étale Galois covering $C^{\prime} \rightarrow C$ with group $G$ such that $J^{\prime}=J \times_{C}$ $C^{\prime}=E \times{ }_{k} C^{\prime}$, for some elliptic curve $E$ over $k$. Since forming $\mathrm{Pic}^{0}$ commutes with base change, the Jacobian associated to the fibration $X^{\prime}=X \times{ }_{C} C^{\prime} / C^{\prime}$ will be $J^{\prime}$. We denote by $\mathcal{X}^{\prime} \rightarrow \mathcal{X}$ the unique lifting of $X^{\prime} \rightarrow X$.

We claim that $\mathcal{X}^{\prime}$ admits an elliptic fibration. To see this, we show that the deformation functors $\mathcal{F} i b_{X^{\prime} / C^{\prime}}$ and $\mathcal{D}$ ef ${ }_{J^{\prime}}$ are isomorphic.

The functor of Jacobian deformations of $J^{\prime}$ is unobstructed (see Corollary 3.3), so we conclude by Proposition 4.1 that $\mathcal{F} i b_{X^{\prime}} / C^{\prime}$ is unobstructed as well. It remains to show that

$$
h^{1}\left(X^{\prime}, \Theta_{X^{\prime}}\right)=\operatorname{dim}\left(\mathcal{F} i b_{X^{\prime} / C^{\prime}}(k[\epsilon])\right) .
$$

Let $g$ denote the genus of $C$. We have $h^{1}\left(X^{\prime}, \Theta_{X^{\prime}}\right) \leq 4 g-2$ by Lemma 5.4. As for $\mathcal{F} i b_{X^{\prime} / C^{\prime}}(k[\epsilon])$, we have $(3 g-3)+1$ dimensions coming from the functor of Jacobian deformations of $J^{\prime}$; namely, $3 g-3$ from the deformations of the $C^{\prime}$, and one dimension coming from $E$. The first cohomology of the Lie algebra $\mathscr{O}_{C^{\prime}}$ of $J^{\prime}$ gives $g$ additional dimensions.

Now, we come back to our deformation $\mathcal{X}$ of $X$. By Proposition 5.3, the fibration $g: \mathcal{X}^{\prime} \rightarrow \mathcal{C}^{\prime}$ is defined in terms of the canonical bundle of $\mathcal{X}^{\prime}$. The action of $G$ an $\mathcal{X}^{\prime}$ induces an action on the canonical model $\mathcal{C}^{\prime}$ of $\mathcal{X}^{\prime}$. For $\sigma \in G$, we get a diagram:

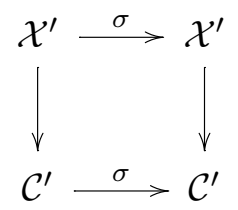

This implies that $g: \mathcal{X}^{\prime} \rightarrow \mathcal{C}^{\prime}$ descends to a fibration $f: \mathcal{X} \rightarrow \mathcal{C}$ on $\mathcal{X}$.

We are going to present some applications of Theorem 5.2. Recall that in Section 3 we constructed a Jacobian elliptic fiber bundle $J$ over some curve of genus 2 over a field of characteristic 3, which was shown to be nonliftable as a Jacobian elliptic fiber bundle. We also gave two examples denoted by $J_{G}$ and $J_{Q}$, showing the same behavior in characteristic 2 . 
THEOREM 5.6. The elliptic fiber bundles $J$ (in characteristic 3) and $J_{G}, J_{Q}$ (in characteristic 2) do not admit a formal lifting to characteristic 0 .

Proof. We already saw that $J, J_{G}$, and $J_{Q}$ cannot be lifted as Jacobian elliptic fiber bundles (see Propositions 3.5 and 3.6). From Proposition 4.1, it follows that the same is true for liftings which are not Jacobian but admit an elliptic fibration. Finally, observe that the base curves in both cases are of genus $g \geq 2$, which implies by Lemma 5.1(iii) that the Kodaira dimensions of $J, J_{G}$, and $J_{Q}$ are 1 . Now, by Theorem 5.2 , we get that every deformation is elliptic.

One can make an interesting remark here. Recall the following conjecture of Oort.

Conjecture. Let $k$ be a field of characteristic $p$, and let $C$ be a curve of genus greater than or equal to 2 over $k$. Let $G$ be a cyclic subgroup of $\operatorname{Aut}(C)$. Then there exists a lifting to characteristic 0 of the pair $(C, G)$.

The conjecture is known to hold if the order of $G$ is not divided by $p^{3}$ (see $[\mathrm{GM}])$. Given a curve $C$ and a cyclic subgroup $G \subset \operatorname{Aut}(C)$, we construct an elliptic surface $X \rightarrow B=C / G$, which has a formal lifting to characteristic 0 if and only if the pair $(C, G)$ is liftable.

Let $E$ be an ordinary elliptic curve over $k$. An action of $G$ on $E$ is given by choosing a point of order $\operatorname{ord}(G)$. We set $X=(E \times C) / G$, where we divide out by the diagonal action of $G$. Note that the action of $G$ on the product is free. The surface $X$ will have an elliptic fibration coming from the projection $E \times C \rightarrow C$. However, this fibration will in general not define an elliptic fiber bundle because the fixed points of the $G$ action on $C$ will give rise to multiple fibers.

THEOREM 5.7. The surface $X$ is formally liftable to characteristic 0 if and only if there exists a lifting $\mathcal{C}$ of $C$ together with a lifting of $G$.

Proof. The "if" part is clear. Assume that there exists a lifting $\mathcal{X}$ of $X$ over some local Artinian ring $\Lambda$ with residue field $k$. Since the covering $X^{\prime}=E \times C \rightarrow X$ given by the quotient map is étale, we find a lifting $\mathcal{X}^{\prime} \rightarrow \mathcal{X}$ which is again Galois with group $G$.

Now, $X^{\prime}$ is an elliptic fiber bundle of Kodaira dimension 1; hence, by Proposition 5.5 we know that $\mathcal{X}^{\prime}$ comes with its canonical fibration $\mathcal{X}^{\prime} \rightarrow \mathcal{C}$ lifting $X^{\prime} \rightarrow C$. Since $\mathcal{C}$ is the canonical model of $\mathcal{X}^{\prime}$, we get an induced $G$-action on $\mathcal{C}$, coming from the $G$-action on $\mathcal{X}$. Since the automorphism 
scheme of a curve of higher genus is unramified, we conclude that the $G$-action on $\mathcal{C}$ is faithful.

\section{§6. Deformations of bielliptic surfaces}

Let $X$ be minimal smooth surface over $k$, of Kodaira dimension 0 and with invariants $b_{1}=b_{2}=2$. Directly from the invariants, we get that the Albanese of $X$ is an elliptic curve. The associated map $f: X \rightarrow \operatorname{Alb}(X)$ is either a smooth elliptic fibration (see [BM, Proposition 5]) or a quasi-elliptic fibration. In the elliptic case, we call $X$ a bielliptic surface.

To keep the presentation streamlined, we first consider the cases where $f$ has a section. From Proposition 3.2, we know that $X$ is given by a quotient

$$
(E \times F) / G \text {, }
$$

where $F \rightarrow \operatorname{Alb}(X)$ is an étale Galois covering with group $G$ (i.e., an étale isogeny), and $G$ acts on $E$ fixing the zero section. Note that this action cannot be trivial, for otherwise $X$ would be an abelian surface. Without loss of generality, we assume that the action of $G$ on $E$ is faithful. Since the fundamental group of an elliptic curve is abelian, $G$ has to be abelian, too. It follows that $G$ equals $\mathbb{Z} / d \mathbb{Z}$, where $d \in\{2,3,4,6\}$. We fix the image of a generator of $G$ in $\operatorname{Aut}_{0}(E)$ and denote it by $\omega$. As a first step, we calculate some invariants of $X$ depending on $p$ and $d$ which are important for the deformation behavior of $X$.

Proposition 6.1. Let $X$ be a bielliptic surface. Denote by $f: X \rightarrow C$ its Albanese map. Assume that $f$ has as section. Write

$$
X=\left(E \times C^{\prime}\right) / G .
$$

If $d$ is not a power of $p$ and $d \neq 2$, we have

$$
h^{0}\left(\Theta_{X}\right)=1, \quad h^{1}\left(\Theta_{X}\right)=1, \quad h^{2}\left(\Theta_{X}\right)=0, \quad h^{1}(C, \operatorname{Lie}(X / C))=0 .
$$

If $d=2$ and $p \neq 2$, we get

$$
h^{0}\left(\Theta_{X}\right)=1, \quad h^{1}\left(\Theta_{X}\right)=2, \quad h^{2}\left(\Theta_{X}\right)=1, \quad h^{1}(C, \operatorname{Lie}(X / C))=0 ;
$$

whereas if $d$ is a power of $p$, it holds that

$$
h^{0}\left(\Theta_{X}\right)=2, \quad h^{1}\left(\Theta_{X}\right)=4, \quad h^{2}\left(\Theta_{X}\right)=2, \quad h^{1}(C, \operatorname{Lie}(X / C))=1 .
$$

Let $Y / C$ be a non-Jacobian bielliptic surface with Jacobian $X / C$. Then we have

$$
h^{i}\left(Y, \Theta_{Y}\right)=h^{i}\left(X, \Theta_{X}\right)
$$


Proof. Since $f$ is smooth, we have an exact sequence

$$
0 \rightarrow \Theta_{X / C} \rightarrow \Theta_{X} \rightarrow f^{*} \Theta_{C} \rightarrow 0
$$

Since the action of $\mathbb{Z} / d \mathbb{Z}$ on $E \times C^{\prime}$ is diagonal, (6.1) is split. Therefore, $\Theta_{X}$ decomposes as

$$
\Theta_{X} \simeq \Theta_{X / C} \oplus f^{*} \Theta_{C}
$$

Since $C$ is an elliptic curve, $f^{*} \Theta_{C} \simeq \mathscr{O}_{X}$. As for $\Theta_{X / C}$, it will be a torsion line bundle of order $\ell$ equal to the order of the induced action of $\mathbb{Z} / d \mathbb{Z}$ on $\Theta_{E}$. To see this, note that $\mathbb{Z} / d \mathbb{Z}$ acts trivially on $\Theta_{E}^{\otimes \ell}$ because the induced action is by roots of unity. Thus, a section of $\Theta_{E}^{\otimes \ell}$ will descend to a section of $\Theta_{X / C}^{\otimes \ell}$. We claim that $\operatorname{ord}\left(\Theta_{X / C}\right)=\ell$, where $d=\ell p^{n}$ with $\ell$ prime to $p$. This is seen as follows.

The action on $\Theta_{E}$ is determined by the action on the $k$-vector space $\operatorname{Lie}(E)$. If $d$ is a power of $p$, this action has to be trivial since $\operatorname{Hom}\left(\mathbb{Z} / p^{n} \mathbb{Z}\right.$, $\left.\mathbb{G}_{m}\right)=0$. To determine the action in general, note that we have a subgroup scheme $H \subset E$ of height 1 such that $\operatorname{Lie}(H)=\operatorname{Lie}(E)$. In fact, the total space of $H$ is given by $\operatorname{Spec}\left(\mathscr{O}_{E} / \mathfrak{m}_{0, E}^{p}\right)$, and $H$ is isomorphic either to $\mu_{p}$ or to $\alpha_{p}$. Because of height 1 , the map given by the Lie functor $\operatorname{Aut}(H) \rightarrow$ $\operatorname{Aut}(\operatorname{Lie}(H))$ is injective. In fact, it will be an isomorphism if we restrict to maps of $p$-Lie algebras (see [Mu, Section 14]).

The group scheme $H$ is of $\operatorname{rank} p$, so if $p>4$ we get $\operatorname{ord}(\omega)=\operatorname{ord}\left(\left.\omega\right|_{H}\right)$ by rigidity (see [KM, Corollary 2.7.3]). If $p=3$ and $d=2$, we know that $\omega$ will act on $\operatorname{Lie}(E)$ as involution. If $d=4$, the same argument applies to $\omega^{2}$.

If $p=2$ and $d=3$, we have $H=\alpha_{2}$ since $j(E)=0$. Assume that $\omega$ induces the identity on $H$. Then the associated trace map $\operatorname{tr}_{\omega}=\mathrm{Id}+\omega+\omega^{2}$ would give multiplication by 3 , which is an isomorphisms on $H$. However, we know that $\operatorname{tr}_{\omega}$ is the zero map on $E$ (see Lemma 6.5 below).

Now it is easy to calculate the invariants. Denote by $\epsilon: C \rightarrow X$ the zero section of $X$. We have $\mathscr{L}=\operatorname{Lie}(X)=\epsilon^{*} \Theta_{X / C}$, and since $f^{*} \operatorname{Lie}(X) \simeq \Theta_{X / C}$, it follows that

$$
\operatorname{ord}(\operatorname{Lie}(X))=\operatorname{ord}\left(\Theta_{X / C}\right) \text {. }
$$

The statement about the cohomology of $\operatorname{Lie}(X)$ follows, since it is a line bundle of degree 0 , and therefore

$$
h^{1}(C, \operatorname{Lie}(X))=h^{0}(C, \operatorname{Lie}(X))
$$


However, the last term is not zero if and only if $\operatorname{Lie}(X)$ is trivial. We also get that

$$
h^{0}\left(X, \Theta_{X}\right)=h^{0}\left(X, \Theta_{X / C}\right)+h^{0}\left(X, f^{*} \Theta_{C}\right)=h^{0}(X, \operatorname{Lie}(X))+1 .
$$

To compute $h^{1}\left(X, \Theta_{X}\right)$, we treat both summands in (6.2) separately. By Lemma 5.1(ii), we have $\Theta_{X / C} \simeq f^{*} R^{1} f_{*} \mathscr{O}_{X} \simeq f^{*} \operatorname{Lie}(X)$. By the projection formula $\left(R^{1} f_{*} f^{*} \mathscr{L} \simeq \mathscr{L}^{\otimes 2}\right)$ and the Leray spectral sequence, we get

$$
0 \rightarrow H^{1}(C, \mathscr{L}) \rightarrow H^{1}\left(X, \Theta_{X / C}\right) \rightarrow H^{0}\left(C, R^{1} f_{*} f^{*} \mathscr{L}\right) \rightarrow 0 .
$$

Thus, we have

$$
h^{1}\left(X, \Theta_{X / C}\right)= \begin{cases}0 & \text { if } \ell>2 \\ 1 & \text { if } \ell=2 \\ 2 & \text { if } \ell=1\end{cases}
$$

For $h^{1}\left(X, f^{*} \Theta_{C}\right)$, we obtain similarly

$$
0 \rightarrow H^{1}\left(C, \Theta_{C}\right) \rightarrow H^{1}\left(X, f^{*} \Theta_{C}\right) \rightarrow H^{0}\left(C, \mathscr{L} \otimes \Theta_{C}\right) \rightarrow 0
$$

Since $\Theta_{C} \simeq \mathscr{O}_{C}$, we find that $h^{1}\left(X, f^{*} \Theta_{C}\right)=2$ if $\mathscr{L}$ is trivial and that $h^{1}\left(X, f^{*} \Theta_{C}\right)=1$ otherwise.

This proves the statement about $h^{1}\left(X, \Theta_{X}\right)$. To compute $h^{2}\left(X, \Theta_{X}\right)$, we just observe that $\chi\left(\Theta_{X}\right)=0$, because $\chi\left(\Theta_{E \times C^{\prime}}\right)=0$.

The statement about a non-Jacobian bielliptic surface $g: Y \rightarrow C$ with Jacobian $X / C$ follows from the expression

$$
R^{1} g_{*} \mathscr{O}_{Y} \simeq \operatorname{Lie}(X / C) \simeq \epsilon^{*} \Theta_{J / C}
$$

and $g^{*}(\mathscr{L})=\Theta_{Y / C}$.

\subsection{The versal families}

Let $X=(E \times F) / G$ be a Jacobian bielliptic surface over $k$. First, we study the deformation functor $\mathcal{J} a c_{X / C}$.

By Proposition 3.2, we know the structure of Jacobian deformations of $X$. They will be of the form $(\mathcal{E} \times \mathcal{F}) / \Gamma$. Here, $\mathcal{E}$ is a deformation of $E$ extending the automorphism $\omega$, and we are going to denote the deformation functor of such pairs by $(E, \omega)$. Likewise, $\mathcal{F}$ is a deformation of $F$ with a torsion point lifting the point of $F$ which appears in the definition of the action of $\Gamma$, and we denote the deformation functor of such pairs by $(F, c)$. 
The functor $\mathcal{J} a c_{X / C}$ is isomorphic to the product of the deformation functors

$$
(E, \omega) \times(F, c) .
$$

To write down a versal family for $\mathcal{J} a c_{X / C}$, we treat the problem separately for both factors.

6.1.1. Deforming elliptic curves with automorphisms. Let $\left(\mathcal{E}^{\text {univ }}, \omega\right) \rightarrow$ $\operatorname{Spec}(R)$ be the universal deformation of $E$ along with its automorphism $\omega$. This functor is indeed pro-representable. Given that a lifting of $\omega$ exists for a given deformation of $E$, then it is unique.

If $\operatorname{ord}(\omega)=2$, then $\omega$ is the involution, which obviously extends to any deformation of $E$. Hence, in that case $R=W[[t]]$.

If $\operatorname{ord}(\omega)>2$, then the $j$-invariant of $\mathcal{E}$ is either 0 or 1728 . If the order is prime to $p$, there are no obstructions against lifting $(E, \omega)$; thus, $R=W$.

We treat the remaining cases. First, assume that $p=2$ and that $d=$ $\operatorname{ord}(\omega)=4$. We know from [JLR, Lemma 1.1] that there is no elliptic curve over $W$ with $j$-invariant 1728 and good reduction. This means that we have to pass to a ramified extension of $W$. We will work over $R=W[i]$, where $i$ is a primitive fourth root of unity. The following curve $\mathcal{E}_{2}$ is taken from [JLR, Section 2.A]:

$$
y^{2}+(-i+1) x y-i y=x^{3}-i x^{2} .
$$

It has $j=1728$ and discriminant $\Delta=11-2 i$ and is therefore of good reduction.

For $p=3$ and $d=3$, again by [JLR, Section $2 \mathrm{~A}$ ] there will be no elliptic curve over $W$ with $j$-invariant 0 and good reduction. So let $R=W[\pi]$, where $\pi^{2}=3$. Consider the elliptic curve $\mathcal{E}_{3}$ given by the Weierstrass equation

$$
y^{2}=x^{3}+\pi x^{2}+x,
$$

whose $j$-invariant is 0 and whose discriminant is $\Delta=-16$. In particular, it has good reduction.

In both cases $(p=2$ or 3$)$, the curve $\mathcal{E}_{p}$ has an automorphism of order 4 or 3 , respectively, since on the generic fiber automorphisms are given by the action of certain roots of unity, and we have chosen the base rings in such a way that they contain the necessary roots. An automorphism of the generic fiber extends to the entire family, and its order will not change when restricting to the special fiber, as can be seen by considering an étale torsion subscheme of sufficiently high order. 
We claim that the elliptic curves over the rings constructed above are the universal families for the deformation problem $(E, \omega)$. This follows from the fact that the respective base rings are the smallest possible extensions of $W$ over which the deformation problem can be solved, and from the fact that an elliptic curve over a strictly Henselian ring is determined by its $j$-invariant.

6.1.2. Deforming elliptic curves with torsion points. Now we treat the second factor. If $p$ does not divide $d$, then a $d$-torsion point lifts uniquely to any deformation of $F$. Therefore, $(F, c)$ is pro-represented by $W[[t]]$.

Assume now that $p$ does divide $d$. By the above, we can assume that $d=p^{n}$. Since $F$ is ordinary, we can use the Serre-Tate theory of local moduli. (For the special case of ordinary elliptic curves, see [KM, Section 8.9].) By this theory, we can represent the deformation functor of $F$ by a pair

$$
\mathcal{F}^{\text {univ }} \rightarrow \operatorname{Spec}(W[[q-1]])
$$

satisfying the following property. For a complete local $W[[q-1]]$-algebra $\Lambda$, consider the pullback

$$
\mathcal{F}=\mathcal{F}^{\text {univ }} \otimes_{W[[q-1]]} \Lambda .
$$

By $\mathbb{Z}\left[q, q^{-1}\right] \rightarrow W\left[[q-1] \rightarrow \Lambda\right.$, we make $\Lambda$ into a $\mathbb{Z}\left[q, q^{-1}\right]$-algebra. There exists a universal group scheme $T$ over $\mathbb{Z}\left[q, q^{-1}\right]$ defined in [KM, Section 8.7] such that

$$
\mathcal{F}\left[p^{\infty}\right] \simeq T\left[p^{\infty}\right] \otimes_{\mathbb{Z}\left[q, q^{-1}\right]} \Lambda
$$

The explicit description of $T$ in [KM, Section 8.7] implies that the sequence

$$
0 \rightarrow \mu_{p^{n}} \rightarrow T\left[p^{n}\right] \otimes \Lambda \rightarrow \mathbb{Z} / p^{n} \mathbb{Z} \rightarrow 0
$$

is split if and only if the image of $q$ in $\Lambda$ has a $p^{n}$ th root. However, (6.3) is split if and only if $c$ lifts to $\mathcal{F}$.

We conclude that $W[[q-1]][\sqrt[p]{q}]$ is a versal hull of the functor $(F, c)$.

Proposition 6.2. The versal hull $R$ of the functor $\mathcal{J} a c_{X / C}$ is given as follows:

\begin{tabular}{|c|c|c|c|}
\hline & $p=2$ & $p=3$ & $p>3$ \\
\hline$d=2$ & $W\left[\left[t_{E}\right]\right] \otimes W[[q-1]][\sqrt[2]{q}]$ & $W\left[\left[t_{E}\right]\right] \otimes W\left[\left[t_{F}\right]\right]$ & $W\left[\left[t_{E}\right]\right] \otimes W\left[\left[t_{F}\right]\right]$ \\
\hline$d=3$ & $W\left[\left[t_{E}\right]\right] \otimes W$ & $W[\pi] \otimes W[[q-1]][\sqrt[3]{q}]$ & $W \otimes W\left[\left[t_{F}\right]\right]$ \\
\hline$d=4$ & $W[i] \otimes W[[q-1]][\sqrt[4]{q}]$ & $W \otimes W\left[\left[t_{F}\right]\right]$ & $W \otimes W\left[\left[t_{F}\right]\right]$ \\
\hline$d=6$ & $W \otimes W[[q-1]][\sqrt[2]{q}]$ & $W[\pi] \otimes W[[q-1]][\sqrt[3]{q}]$ & $W \otimes W\left[\left[t_{F}\right]\right]$ \\
\hline
\end{tabular}


It is easy to read off and interpret the dimension of the tangent space of the deformation functor. For example, in the case where $p=3$ and $d=3$, we have $\operatorname{dim}(W[\pi] \otimes W[[q-1]][\sqrt[3]{q}], k[\epsilon])=3$. There is one dimension coming from the deformations of $F$, and the rest is due to relations coming from obstructions. As explained before, we have $h^{1}\left(X, \Theta_{X}\right)=4$ in this case, so one dimension is still missing.

To account for this missing dimension, we have to study all deformations of $X$, not just the Jacobian ones. This is settled by Theorem 4.4. Observe that in all the cases, we have

$$
h^{1}\left(X, \Theta_{X}\right)-\operatorname{dim}\left(\mathcal{J} a c_{X / C}(k[\epsilon])\right)=h^{1}(C, \operatorname{Lie}(X / C)) .
$$

Therefore, $\operatorname{dim}(\mathcal{F} i b(k[\epsilon]))=h^{1}\left(X, \Theta_{X}\right)$, and it makes sense to ask if the absolute deformation functor of $X$ is isomorphic to $\mathcal{F} i b_{X / C}$. In the next section, we will see that this is indeed the case.

\subsection{Classification of deformations}

The most important step to classify deformations of bielliptic surfaces is to show that for a bielliptic surface $X / C$ over $k$, the functors $\mathcal{F} i b_{X / C}$ and Def ${ }_{X}$ are isomorphic.

Denote by $J \rightarrow C$ the Jacobian of $X \rightarrow C$. If $d$ is not a power of $p$, the claim follows already, since in that case $\mathcal{J} a c_{J / C}$ is unobstructed and has the right tangent dimension. Hence, we get a chain of isomorphisms

$$
\mathcal{J} a c_{X / C} \simeq \mathcal{F} i b_{X / C} \simeq \mathcal{D} e f_{X}
$$

In the case where $d$ is a power of $p$, we have to work with the étale covering of $X$. This is more difficult than in the Kodaira dimension 1 case, because the étale cover of the reduction is an elliptic abelian surface, and not every deformation of the covering admits a fibration.

To understand the deformation theory of abelian surfaces, we use $p$-divisible groups. For the reader's convenience, we repeat some basic definitions and facts. Let $p$ be a prime number, and let $S$ be a scheme. A sheaf of groups for the $f p p f$-topology is called a $p$-divisible group, if $G$ is $p$-divisible and $p$-primary, that is,

$$
G=\underset{\longrightarrow}{\lim } G\left[p^{n}\right]
$$

and the groups $G\left[p^{n}\right]$ are finite flat group schemes over $S$. (See [Gr3], where $p$-divisible groups go by the name "Barsotti-Tate groups.") The main examples which we are in fact interested in are $p$-divisible groups associated with 
abelian schemes. For an abelian $S$-scheme $A$, we set

$$
A\left[p^{\infty}\right]=\underset{\lim }{\longrightarrow} A\left[p^{n}\right]
$$

The deformation theory of abelian schemes is controlled by $p$-divisible groups. To be precise, let $R$ be a ring in which $p^{N}=0$. For a nilpotent ideal $I \subset R$, we define the category $\mathcal{T}$ of triples:

$$
(A, \mathcal{G}, \epsilon)
$$

where $A$ is an abelian scheme over $R / I, \mathcal{G}$ is a $p$-divisible group over $R$, and $\epsilon$ is an isomorphism $\mathcal{G} \otimes_{R} R / I \simeq A\left[p^{\infty}\right]$. Now we have the theorem of Serre and Tate.

Theorem 6.3 ([K, Theorem 1.2.1]). There is an equivalence between $\mathcal{T}$ and the category of abelian schemes over $R$ given by

$$
\mathcal{A} \mapsto\left(\mathcal{A} \otimes_{R} R / I, \mathcal{A}\left[p^{\infty}\right], \text { natural } \epsilon\right) .
$$

We will use the following statement to understand the lifting behavior of morphisms of the latter.

Lemma 6.4 ([K, Lemma 1.1.3]). Let $\mathcal{G}$ and $\mathcal{H}$ be p-divisible groups over $R$. Assume that $I^{\nu+1}=0$. Let $G$ and $H$ denote their restrictions to $\operatorname{Spec}(R / I)$. Then the following hold.

(i) The groups $\operatorname{Hom}_{R}(\mathcal{G}, \mathcal{H})$ and $\operatorname{Hom}_{R / I}(G, H)$ have no p-torsion.

(ii) The reduction map $\operatorname{Hom}_{R}(\mathcal{G}, \mathcal{H}) \rightarrow \operatorname{Hom}_{R / I}(G, H)$ is injective.

(iii) For any homomorphism $f: G \rightarrow H$, there exists a unique homomorphism $\phi_{\nu}$ lifting $\left[p^{\nu}\right] \circ f$.

(iv) In order for $f$ to lift to a homomorphism $f: \mathcal{G} \rightarrow \mathcal{H}$, it is necessary and sufficient for the homomorphism $\phi_{\nu}$ to annihilate $\mathcal{G}\left[p^{\nu}\right]$.

In the course of the proof, we will use the following lemma.

Lemma 6.5. Let $\mathcal{E}$ be an elliptic scheme over a base scheme $\mathcal{S}$. Let $\Omega$ be an automorphism of $\mathcal{E}$ of order $d$. We consider the trace map

$$
\operatorname{tr}_{\Omega}=\operatorname{Id}+\Omega+\cdots+\Omega^{d-1} .
$$

Then $\operatorname{tr}_{\Omega}$ gives the zero map on $\mathcal{E}$. 
Proof. We can prove the statement fiberwise. So assume that $S$ is the spectrum of a field. It holds that

$$
(\operatorname{Id}-\Omega) \circ \operatorname{tr}_{\Omega}=\mathrm{Id}-\Omega^{d}=0 .
$$

However, $\mathrm{Id}-\Omega$ is surjective; hence, $\operatorname{tr}_{\Omega}=0$ follows.

Proposition 6.6. Let $f: X \rightarrow C$ be a Jacobian bielliptic surface over $k$. Then $f$ extends to any deformation $\mathcal{X}$ of $X$ over $\Lambda \in \mathcal{A} \lg _{W}$.

Proof. We have an étale Galois cover $A=E \times F$ of $X$. The Galois group is isomorphic to $\Gamma=\mathbb{Z} / d \mathbb{Z}$. The action on $E$ is by an automorphism $\omega$, fixing the zero section. For a deformation $\mathcal{X}$ of $X$, we get a diagram

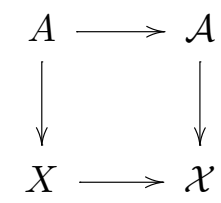

where the right-hand column is the unique lifting of the left. By [MFK, Theorem 6.14], we can give $\mathcal{A}$ a structure of an abelian scheme, extending the group structure on $A$. Now the strategy is as follows. First, we show that $\mathcal{A}$ has an automorphism $\Omega$ lifting $\operatorname{Id} \times \omega$. Then we study the action of $\Omega$ on the $p$-divisible group $\mathcal{A}\left[p^{\infty}\right]$, and we use the trace map defined by $\Omega$ to lift the projection $E\left[p^{\infty}\right] \times F\left[p^{\infty}\right] \rightarrow F\left[p^{\infty}\right]$. This lifting will descend to the desired lifting of the fibration $f$ on $X$.

Denote the image of the generator $1 \in \Gamma$ in $\operatorname{Aut}(\mathcal{A})$ by $\sigma$. Note that $\sigma$ does not necessarily fix the zero section. We study its action on $\mathcal{A}$. Set $c=\sigma(0) \in \mathcal{A}(\operatorname{Spec}(\Lambda))$, and denote by $t_{-c}$ the morphism given by translation on $-c$. We set

$$
\Omega=\sigma \circ t_{-c} \quad \text { and } \quad \Omega^{\prime}=t_{-c} \circ \sigma .
$$

Both maps fix the zero section of $\mathcal{A}$ and are therefore group automorphisms of $\mathcal{A}$. Furthermore, they lift the automorphism $\operatorname{Id} \times \omega$ of $E \times F$, which implies that $\Omega=\Omega^{\prime}$ since the lift of an automorphism is unique.

This means that $\Omega$ and $t_{c}$ commute, and since $\sigma$ and $\Omega$ are of order $d$, we get that $c$ is a torsion point of order $d$, which lifts the action of $\Gamma$ by translation on $F$.

To proceed with the proof, we pass to the category of $p$-divisible groups, as explained in Theorem 6.3. Our aim is to lift the second projection

$$
\mathrm{pr}_{2}: E\left[p^{\infty}\right] \times F\left[p^{\infty}\right] \rightarrow F\left[p^{\infty}\right] .
$$


We know that there exists some integer $N$ such that there exists a unique lift $\phi_{N}$ of $[N] \circ \operatorname{pr}_{2}$ (Lemma 6.4). We compare $\phi_{N}$ with the trace $\operatorname{tr}_{\Omega}$ defined by $\Omega$. The restriction $\overline{\operatorname{tr}_{\Omega}}$ of $\operatorname{tr}_{\Omega}$ to $A\left[p^{\infty}\right]$ gives the map

$$
[d] \circ \operatorname{pr}_{2}: A\left[p^{\infty}\right] \rightarrow F\left[p^{\infty}\right]=\operatorname{Im}\left(\overline{\operatorname{tr}_{\Omega}}\right)
$$

because $\operatorname{tr}_{\Omega}$ is multiplication by $d$ on the factor $F\left[p^{\infty}\right]$ and the zero map on the factor $E\left[p^{\infty}\right]$ (see Lemma 6.5). Now, we get that $[d] \circ \phi_{N}$ is a lift of $[N] \circ \overline{\operatorname{tr}_{\Omega}}$. Again, because an endomorphism has at most one lifting, it follows that

$$
[d] \circ \phi_{N}=[N] \circ \operatorname{tr}_{\Omega} .
$$

Factoring out by $\mathcal{A}[N]$, we see that $\operatorname{tr}_{\Omega}$ is a lift of $[d] \circ\left[\mathrm{pr}_{2}\right]$. It remains to show that $\mathcal{A}[d]$ lies in the kernel of $\operatorname{tr}_{\Omega}$. To see this, we consider the exact sequence of finite flat group schemes

$$
0 \rightarrow \mathcal{A}[d]^{0} \rightarrow \mathcal{A}[d] \rightarrow \mathcal{A}[d]^{\mathrm{et}} \rightarrow 0 .
$$

We first show that $\operatorname{tr}_{\Omega}\left(\mathcal{A}[d]^{0}\right)=0$. Again, we have an exact sequence

$$
0 \rightarrow \mathcal{A}[d]^{\text {mult }} \rightarrow \mathcal{A}[d]^{0} \rightarrow \mathcal{A}[d]^{\mathrm{bi}} \rightarrow 0 .
$$

The outer groups denote the multiplicative part and the bi-infinitesimal part, respectively. The category of multiplicative groups schemes is dual to the category of étale group schemes via Cartier duality; thus, endomorphisms lift uniquely, and we get $\operatorname{tr}_{\Omega}\left(\mathcal{A}[d]^{\text {mult }}\right)=0$. Now, we consider the sequence of $p$-divisible groups

$$
0 \rightarrow \mathcal{A}\left[p^{\infty}\right]^{\text {mult }} \rightarrow \mathcal{A}\left[p^{\infty}\right]^{0} \rightarrow \mathcal{A}\left[p^{\infty}\right]^{\mathrm{bi}} \rightarrow 0 .
$$

Since $\Omega$ maps $\mathcal{A}\left[p^{\infty}\right]^{\text {mult }}$ into itself, we get an induced action of $\Omega$ on $\mathcal{A}\left[p^{\infty}\right]^{\text {bi }}$, and in particular, $\operatorname{tr}_{\Omega}$ descends to $\mathcal{A}\left[p^{\infty}\right]^{\text {bi }}$. If this group is nontrivial, it is a lift of $E\left[p^{\infty}\right]$ on which $\operatorname{tr}_{\Omega}$ is zero. Again by uniqueness of lifts, we get $\operatorname{tr}_{\Omega}\left(\mathcal{A}[d]^{\mathrm{bi}}\right)=0$.

We saw that $\operatorname{tr}_{\Omega}\left(\mathcal{A}[d]^{0}\right)=0$, and it remains to show that $\operatorname{tr}_{\Omega}\left(\mathcal{A}[k]^{\text {ét }}\right)=0$. However, this is clear, since we deal with étale group schemes. We conclude that $\mathrm{pr}_{2}$ extends to $\mathcal{X}$.

So far, we have treated only Jacobian bielliptic surfaces. But the nonJacobian cases are mostly trivial. Consulting the table of bielliptic surfaces in $[\mathrm{BM}]$, we see that the Tate-Safarevič group is trivial if the associated Jacobian has obstructed deformations, except in one case in characteristic 2. 
To construct this surface, let $E$ and $F$ be ordinary elliptic curves over $k$ with $p=2$. We set $A=(E \times F) / \mu_{2}$, where $\mu_{2}$ is the subgroup scheme embedded diagonally into

$$
(E \times F)[2]^{0} \simeq \mu_{2} \times \mu_{2}
$$

The quotient $A$ is an abelian surface which does not split into a product.

Let $c$ be a nontrivial 2-torsion point of $F$. The action on $E \times F$, given by $(x, y) \mapsto(x+c,-y)$, commutes with the diagonal action of $\mu_{2}$ and thereby descends to a $\mathbb{Z} / 2 \mathbb{Z}$-action on $A$. The bielliptic surface $X$ we are interested in is now given by $A /(\mathbb{Z} / 2 \mathbb{Z})$. The Jacobian of $X$ is clearly $J=$ $(E \times F) /(\mathbb{Z} / 2 \mathbb{Z})$.

Now let $\mathcal{X}$ be a deformation of $X$. Once more we have a diagram:

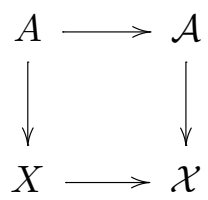

We claim that $\mathcal{A}$ admits a lifting of the elliptic fibration $f: A \rightarrow F / \mu_{2}$. We have an exact sequence

$$
0 \rightarrow \mathcal{A}\left[p^{\infty}\right]^{\text {tor }} \rightarrow \mathcal{A}\left[p^{\infty}\right] \rightarrow \mathcal{A}\left[p^{\infty}\right]^{\text {ét }} \rightarrow 0 .
$$

The morphism $A\left[p^{\infty}\right]^{\text {ét }} \rightarrow F\left[p^{\infty}\right]^{\text {ét }}$ induced by $f$ lifts uniquely to

$$
\varphi: \mathcal{A}\left[p^{\infty}\right]^{\text {ét }} \rightarrow \mathcal{F}\left[p^{\infty}\right]^{\text {ét }}
$$

since we are dealing with étale group schemes. Denote by $\mathcal{B}$ the $p$-divisible group obtained by pushout of (6.4) along $\varphi$. We still have $\mathcal{A}\left[p^{\infty}\right]^{\text {tor }} \subset \mathcal{B}$, and inside $\mathcal{A}\left[p^{\infty}\right]^{\text {tor }}$ is contained the kernel of the unique lift of $A^{\text {tor }} \rightarrow F\left[p^{\infty}\right]^{\text {tor }}$. Dividing out $\mathcal{B}$ by that kernel, we obtain a lifting of $f$.

As in the proof of Proposition 6.6, we see that $f$ descends to $\mathcal{X}$. Therefore, $\mathcal{X}$ is elliptic. To sum up, we have the following theorem.

THEOREM 6.7. Every deformation $X$ of a bielliptic surface $X$ induces a lifting of the elliptic fibration $X \rightarrow C=\operatorname{Alb}(X)$.

Next, we show that a versal deformation of a bielliptic surface is algebraizable. 
Proposition 6.8. Let $X$ be a bielliptic surface over $k$. Denote by $\mathcal{X}^{\text {vers }} \rightarrow$ $\operatorname{Spf}(R)$ a formal versal family of $\mathcal{D}$ ef ${ }_{X}$. Then there exists a projective scheme $\overline{\mathcal{X}}$ over $R$ such that $\mathcal{X}^{\text {vers }}$ is the completion of $\overline{\mathcal{X}}$ at the special fiber.

Proof. For an arbitrary deformation $\mathcal{X}$ of $X$, denote by $\mathcal{A} \rightarrow \mathcal{X}$ the unique lifting of the abelian covering $A \rightarrow X$. In the proof of Proposition 6.6 we saw that the abelian scheme $\mathcal{A}$ has an automorphism $\Omega$ lifting the automorphism $\omega \times \operatorname{Id}$ of $E \times F$.

The automorphism of $\mathcal{X}$, obtained from $\Omega$ by descent, will again be denoted by $\Omega$. Now $\Omega$ is a $\mathcal{C}$-automorphism of $\mathcal{X}$; that is, its action is confined to the fibers of the fibration.

We claim that the fixed locus of $\Omega$ is flat over $\mathcal{C}$. Every closed point $x \in \mathcal{C}$ has an étale neighborhood $\mathcal{U} \rightarrow \mathcal{C}$ such that the pullback

$$
\mathcal{X}_{\mathcal{U}}=\mathcal{X} \times{ }_{\mathcal{C}} \mathcal{U}
$$

can be given in the structure of an abelian scheme in such a way that the base change of $\Omega$ to $\mathcal{X}_{\mathcal{U}}$ becomes a group automorphism. We consider the endomorphism $\Omega-$ Id of $\mathcal{X}_{\mathcal{U}}$. It is a surjective map of abelian schemes and therefore flat by [MFK, Lemma 6.12]. In particular, its kernel, that is, the fixed locus of $\Omega$, is flat over $\mathcal{U}$.

Thus, we have found a relative Cartier divisor of $\mathcal{X} \rightarrow \mathcal{C}$. Its degree can be computed on the reduction. It equals the order of the subgroup scheme of $E$ fixed by $\omega$. In particular, it is positive, which means that $\mathcal{Z}$ is a relatively ample divisor for $\mathcal{X} \rightarrow \mathcal{C}$.

Now denote by $\mathcal{X}^{\text {vers }} \rightarrow \operatorname{Spf}(R)$ a versal family of $\mathcal{F} i b_{X / C}$. It is a formal scheme over the hull of the deformation functor $\mathcal{F} i b_{X / C}$, and we conclude from Theorem 6.7 that it admits an elliptic fibration $F: \mathcal{X}^{\text {vers }} \rightarrow \mathcal{C}$ lifting $X \rightarrow C$.

Denote by $\mathfrak{m}$ the maximal ideal of $R$, and set $X_{n}=\mathcal{X}^{\text {vers }} \otimes_{R} R / \mathfrak{m}^{n+1}$. The construction of $\mathcal{Z}$ gives rise to a compatible system of relatively ample invertible sheaves $\mathscr{O}_{X_{n}}\left(Z_{n}\right)$. Tensoring with the invertible sheaf coming from the divisor of a fiber of $\mathcal{X}^{\text {vers }} \rightarrow \mathcal{C}$, we obtain a system of ample line bundles $\mathscr{H}_{n}$. Thus, by Grothendieck's algebraization theorem (see [I, Theorem 4.10]), we conclude that $\mathcal{X}^{\text {vers }}$ is the completion of some projective scheme $\overline{\mathcal{X}^{\text {vers }}}$ over $\operatorname{Spec}(R)$.

The above proposition helps us to answer another natural question. The surface $X$ is called bielliptic because it has two transversal elliptic fibrations: the smooth one, denoted by $f$, coming from the projection $E \times F \rightarrow F$, and 
a second one, denoted by $g$, with base curve $\mathbb{P}_{k}^{1}$, coming from $E \times F \rightarrow E$. We saw that the first fibration is preserved under deformation, but what about the second one?

Proposition 6.9. Let $X$ be a bielliptic fibration. Then every deformation $\mathcal{X}$ of $X$ extends both elliptic fibrations.

Proof. We are going to show that the versal deformation $\mathcal{X}^{\text {vers }} \rightarrow \operatorname{Spf}(R)$ admits an extension of $g$. Then the claim follows by versality.

Denote by $K$ the fraction field of $R$. We can use surface theory to analyze the generic fiber $\overline{\mathcal{X}}_{K}$ of the algebraization $\overline{\mathcal{X}}$ of $\mathcal{X}^{\text {vers }}$. Denote by $\mathscr{L}=\mathscr{O}_{\overline{\mathcal{X}}}(\mathcal{Z})$ the line bundle associated to the divisor $\mathcal{Z}$, constructed in the proof of Proposition 6.8. The canonical bundle of $\overline{\mathcal{X}}_{K}$ has self-intersection number 0 . It follows that the line bundle $\mathscr{L}_{K}^{\otimes m}$ gives rise to an elliptic fibration $g^{\prime}: \overline{\mathcal{X}}_{K} \rightarrow \mathbb{P}_{K}^{1}$, if we choose $m$ sufficiently big (see [B, Theorem 7.11]).

Since $\overline{\mathcal{X}}$ is proper and normal, we can extend $g^{\prime}$ to a rational map $g^{\prime}: \overline{\mathcal{X}} \rightarrow$ $\mathbb{P}_{R}^{1}$ which is defined on a nonempty open subset intersecting the special fiber. Now there are sections $s_{1}, s_{2}: \operatorname{Spec}(R) \rightarrow \mathbb{P}_{R}^{1}$ whose associated closed subschemes are disjoint and that do lie inside the image of $g^{\prime}$. Taking the closures of the inverse images of those sections under $g^{\prime}$, we get two divisors $\mathcal{G}_{1}$ and $\mathcal{G}_{2}$ in $\overline{\mathcal{X}}$ that have disjoint specializations on a nonempty open subset of the special fiber (namely, the locus where $g^{\prime}$ is defined).

We claim that their reductions $G_{1}$ and $G_{2}$ are irreducible (and hence disjoint). The group of divisors of $X$ modulo numerical equivalence is generated by two classes $F$ and $G$, where $F$ is a fiber class of $f$ and where $G$ is a fiber class of $g$. The intersection numbers are

$$
F \cdot F=0, \quad F \cdot G>0, \quad G \cdot G=0 .
$$

In particular, there are no effective divisors on $X$ with negative selfintersection. It follows that the specialization of a curve of canonical type is again of canonical type. However, every curve of canonical type on $X$ is irreducible; hence, the claim follows.

Considering the global sections of $\mathscr{L}^{\otimes m}$ associated to the effective divisors $\mathcal{G}_{1}$ and $\mathcal{G}_{2}$, we find that $\mathscr{L}^{\otimes m}$ is globally generated. It follows that the map given by $\mathscr{L}^{\otimes m}$ is in fact a morphism, lifting $g$.

We illustrate the theorem by looking at a special case. Let $k$ be of characteristic 3, and let $X$ denote the Jacobian bielliptic surface of index $d=3$ over $k$. What does the fiber $\mathcal{X}_{\eta}$ of the versal family of $X$ over the generic 
point of the base look like? The smooth fibration with elliptic base curve does not have a section. The three sections which appear when we do base change with the algebraic closure of $\eta$ do not descend to $\mathcal{X}_{\eta}$. Instead, we have a multisection of degree 3 .

There is an explicit construction of a bielliptic surface with $d=3$ over $\mathbb{Q}$, which shows the same behavior. It was given in [BS, Theorem 1] as a counterexample to the Hasse principle which cannot be explained by the Manin obstruction.

Acknowledgments. This article is a part of my Ph.D. dissertation. I am indebted to my adviser Stefan Schröer for introducing me to liftability questions and for helpful discussions. I would also like to thank William Lang, Christian Liedtke, Matthias Schütt, Philipp Gross, and one of the referees for inspiring conversations and hints and for highlighting some mistakes in a previous version of this article.

\section{REFERENCES}

[ABD+] M. Artin, J. E. Bertin, M. Demazure, P. Gabriel, A. Grothendieck, M. Raynaud, and J.-P. Serre, Schémas en groupes, Fasc. 7: Exposés 23 à 26, Séminaire de Géométrie Algébrique de l'Institut des Hautes Études Scientifiques 1963/64 (SGA 3), 1st ed., Inst. Hautes Études Sci., Paris, 1965/1966. MR 0207710.

[B] L. Bădescu, Algebraic Surfaces, Universitext, Springer, New York, 2001. MR 1805816.

[BS] C. L. Basile and A. Skorobogatov, "On the Hasse principle for bielliptic surfaces" in Number Theory and Algebraic Geometry, London Math. Soc. Lecture Note Ser. 303, Cambridge University Press, Cambridge, 2003, 31-40. MR 2053453.

[BM] E. Bombieri and D. Mumford, "Enriques' classification of surfaces in char. $p$, II" in Complex Analysis and Algebraic Geometry, Iwanami Shoten, Tokyo, 1977, 23-42. MR 0491719.

[GM] B. Green and M. Matignon, Liftings of Galois covers of smooth curves, Compos. Math. 113 (1998), 237-272. MR 1645000. DOI 10.1023/A:1000455506835.

[Gr1] A. Grothendieck, Éléments de géométrie algébrique, II: Étude globale élémentaire de quelques classes de morphismes, Publ. Math. Inst. Hautes Études Sci. 8, 1961. MR 0217084.

[Gr2] - Revêtements étales et groupe fondamental, Fasc. II: Exposés 6, 8 à 11, Séminaire de Géométrie Algébrique 1960/1961, Inst. Hautes Études Sci., Paris, 1963. MR 0217088.

[Gr3] , Groupes de Barsotti-Tate et cristaux de Dieudonné, Sémin. Math. Supér. 45, Université de Montréal, Montreal, 1974. MR 0417192.

[I] L. Illusie, "Grothendieck's existence theorem in formal geometry" in Fundamental Algebraic Geometry, Math. Surveys Monogr. 123, Amer. Math. Soc., Providence, 2005, 179-233. MR 2223409.

[JLR] T. J. Jarvis, W. E. Lang, and J. R. Ricks, Integral models of extremal rational elliptic surfaces, Comm. Algebra 40 (2012), 3867-3883. 
[KU] T. Katsura and K. Ueno, On elliptic surfaces in characteristic p, Math. Ann. 272 (1985), 291-330. MR 0799664. DOI 10.1007/BF01455561.

[K] N. M. Katz, "Serre-Tate local moduli" in Algebraic Surfaces (Orsay, 1976-1978), Lecture Notes in Math. 868, Springer, Berlin, 1981, 138-202. MR 0638600.

[KM] N. M. Katz and B. Mazur, Arithmetic Moduli of Elliptic Curves, Ann. of Math. Stud. 108, Princeton University Press, Princeton, 1985. MR 0772569.

[Kl] S. L. Kleiman, "The Picard scheme" in Fundamental Algebraic Geometry, Math. Surveys Monogr. 123, Amer. Math. Soc., Providence, 2005, 235-321. MR 2223410.

[L] W. E. Lang, Examples of liftings of surfaces and a problem in de Rham cohomology, Compos. Math. 97 (1995), 157-160. MR 1355122.

[LLR] Q. Liu, D. Lorenzini, and M. Raynaud, Néron models, Lie algebras, and reduction of curves of genus one, Invent. Math. 157 (2004), 455-518. MR 2092767. DOI 10. 1007/s00222-004-0342-y.

[M] J. S. Milne, Étale Cohomology, Princeton Math. Ser. 33, Princeton University Press, Princeton, 1980. MR 0559531.

[Mu D. Mumford, Abelian Varieties, Tata Inst. Fund. Res. Stud. Math. 5, Oxford University Press, London, 1970. MR 0282985.

[MFK] D. Mumford, J. Fogarty, and F. Kirwan, Geometric Invariant Theory, 3rd ed., Ergeb. Math. Grenzgeb. (2) 34, Springer, Berlin, 1994. MR 1304906. DOI 10. 1007/978-3-642-57916-5.

[PS] A. Pacheco and K. F. Stevenson, Finite quotients of the algebraic fundamental group of projective curves in positive characteristic, Pacific J. Math. 192 (2000), 143-158. MR 1741024. DOI 10.2140/pjm.2000.192.143.

[R] M. Raynaud, Faisceaux amples sur les schémas en groupes et les espaces homogènes, Lecture Notes in Math. 119, Springer, Berlin, 1970. MR 0260758.

[S] M. Schlessinger, Functors of Artin rings, Trans. Amer. Math. Soc. 130 (1968), 208-222. MR 0217093.

[Si] J. H. Silverman, The Arithmetic of Elliptic Curves, 2nd ed., Grad. Texts in Math. 106, Springer, Dordrecht, 2009. MR 2514094. DOI 10.1007/978-0-387-09494-6.

Mathematisches Institut

Heinrich-Heine-Universität

D-40225 Düsseldorf

Germany

partsch@math.uni-duesseldorf.de 